\title{
Automated Microwave Tomography (MWT) Image Segmentation: State-of-the-Art Implementation and Evaluation
}

\author{
Yuchong Zhang \\ Chalmers University of \\ Technology \\ Gothenburg, Sweden \\ yuchong@chalmers.se \\ Rahul Yadav \\ University of Eastern \\ Finland \\ Kuopio, Finland \\ rahuly@uef.fi
}

\author{
Yong $\mathrm{Ma}$ \\ Ludwig Maximilian \\ University of Munich \\ Munich, Germany \\ yong.ma@ifi.Imu.de \\ Morten Fjeld \\ Chalmers University of \\ Technology \\ Gothenburg, Sweden \\ fjeld@chalmers.se
}

\author{
Adel Omrani \\ Karlsruhe Institute of \\ Technology \\ Karlsruhe, Germany \\ adel.hamzekalaei@kit.edu \\ Marco Fratarcangeli \\ Chalmers University of \\ Technology \\ Gothenburg, Sweden \\ marcof@chalmers.se
}

\begin{abstract}
Inspired by the high performance in image-based medical analysis, this paper explores the use of advanced segmentation techniques for industrial Microwave Tomography (MWT). Our context is the visual analysis of moisture levels in porous foams undergoing microwave drying. We propose an automatic segmentation technique-MWT Segmentation based on $K$-means (MWTS-KM) and demonstrate its efficiency and accuracy for industrial use. MWTS-KM consists of three stages: image augmentation, grayscale conversion, and $K$-means implementation. To estimate the performance of this technique, we empirically benchmark its efficiency and accuracy against two well-established alternatives: Otsu and $K$-means. To elicit performance data, three metrics (Jaccard index, Dice coefficient and false positive) are used. Our results indicate that MWTS-KM outperforms the well-established Otsu and $K$-means, both in visually observable and objectively quantitative evaluation.
\end{abstract}

\section{Keywords}

Image Segmentation, Otsu, $K$-means, Microwave Tomography.

\section{INTRODUCTION}

\subsection{Image segmentation and MWT}

Image segmentation is one of the most commonly used methods to classify the pixels of an image correctly in decision oriented applications. Due to its capability for distinguishing various features [1], it is able to divide an image into a number of discrete regions such that the pixels have high similarity within and high contrast between regions [2]. It has been well proven that segmentation is widely beneficial to a range of tomographic systems especially Computed Tomography (CT) $[3,4,5,6,7]$. For example, applications like segmenting different tissue types-e.g., lungs, kidneys,

\footnotetext{
Permission to make digital or hard copies of all or part of this work for personal or classroom use is granted without fee provided that copies are not made or distributed for profit or commercial advantage and that copies bear this notice and the full citation on the first page. To copy otherwise, or republish, to post on servers or to redistribute to lists, requires prior specific permission and/or a fee.
}

livers-in a CT image are reliable for accurate and precise diagnoses. There are many application areas for tomographic systems. For instance, while the adoption of image segmentation has been successful on a Microwave Tomography (MWT) system, this is mainly concerning medical usage. This paper focuses on leveraging state-of-the-art segmentation methods to MWT images for specific physical attribute detection in an industrial process setting.

MWT is a non-ionizing imaging technique that provides a quantitative image of the dielectric profile of the object of interest $(\mathrm{OI})[8,9]$. In this paper, we focus on using MWT to observe a specialized industrial process-microwave drying for porous foams. An important physical parameter-moisture level of the foam-is measured throughout the whole drying process. The moisture level is regarded as the measure of a complete drying process. Depending on the imaging techniques used, a reconstructed tomographic images can display either the location of the moisture through the foam as an OI or a map of the dielectric properties of the moisture value both commonly referred to as 
MWT techniques $[10,11]$. In our setup, the foam to be dried is measured and characterized by MWT sensors processed by a series of imaging techniques. After image reconstruction, the dielectric properties of the foam represents a moisture level, depicted in Figure 1, which is an example of reconstructed images using the MWT system. Hence, with the aid of mixing rule techniques [12], an ad hoc mapping is defined to obtain the moisture level of the foam.

After a drying process, some regions of the foam may not be completely dry. It is obvious that dry parts have less moisture, hence the amplitude (moisture or dielectric level) of these areas is lower than in the non-dry regions. The difference between dry and non-dry is represented by allocating different colors to each region (Figure 1) where the blue parts stand for good/low-moisture and the yellow parts stand for bad/high-moisture. Dark blue parts are drier than yellow areas. The colours become more yellow when there is more moisture. It is critical for operators and practitioner to precisely assess the low moisture areas of the material so as to gauge the success of a drying process.

There are some efficient MWT systems that produce high-quality images for industrial processes $[11,9]$. In our drying process, the measurement of the foams is also performed by MWT, whose detailed design is beyond the scope of this paper. We address the segmentation for visualizing the blue regions representing low moisture. Thus, we propose an automatic segmentation method called MWT Segmentation based on $K$-means (MWTS-KM) for processing the images reconstructed by an MWT in our experiment. This algorithm is fully automatic and the user only has a verification role [1]. Next, we report on how we carried out experiments in practical settings to validate the proposed segmentation method, including how we successfully validated its robustness, performance and reliability.

\subsection{Contributions}

As the full version of our recently published work [13], this paper possesses two main outcomes. Firstly, we propose to visualize the moisture area, more precisely the low moisture area for porous foams using image segmentation methods on MWT images, on the premise that many segmentation methods have demonstrated satisfactory utilization in tomographic systems but not desirable success in the context of MWT. Secondly, a state-of-the-art MWTS-KM segmentation method is proposed to visualize the low moisture area of an image. As a novel integrated algorithm, it is compared with two alternative segmentation algorithms that have been used widely in related application areas.

The organization of this paper is as follows. The related works section briefly presents parts of the current

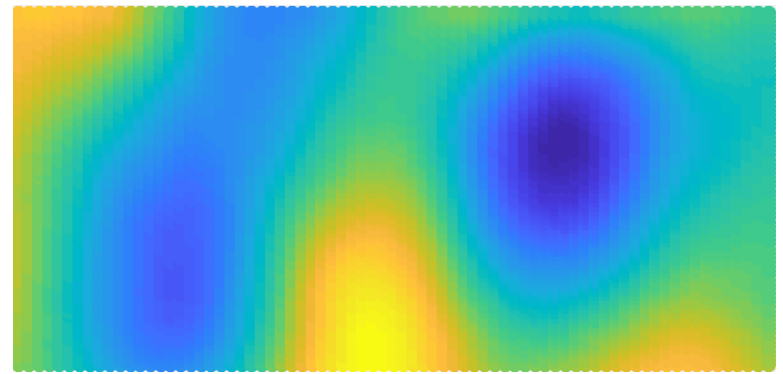

Figure 1: Example of an MWT image (input image) in our study. Blue means lower moisture, yellow means higher moisture.

research situation in segmentation methods used in tomographic images; mostly from the medical application areas. The proposed algorithm and another two common algorithms for comparison are presented in Section 3. Section 4 elaborates the basic experimental setup and the results including evaluation. Discussions and conclusions are given in Sections 5 and 6 respectively.

\section{RELATED WORK}

Significant research efforts have been invested into the topic of tomographic image segmentation and related areas of application. Sharma et al. [14] provided a review of a set of automated segmentation methods ranked by applicability and suitability in the context of tomographic images; especially CT images. Shoaib et al. [15] used the Otsu algorithm [16] to propose a method including thresholding to prove a successful segmentation in lungs using CT. Likewise, Dorgham [1] deployed an automatic segmentation method on the basis of GrabCut [17] to detect human body Regions of Interest (RoI) from CT images. Wu et al. [18] also proposed an automatic segmentation algorithm named AUGC in ultrasound tomography images for breast cancer screening and pathological quantification. This method was also established on GrabCut as well, addressing incomplete labeling and speeding up multicore parallel programming. Moreover, Jose et al. [4] effectively detected and identified the exact location of a brain tumor through $K$-means clustering and fuzzy cmeans algorithms by segmentation CT images. Sheppard et al. [19] utilized a combination algorithm containing active contours [20] and watershed transformation [21] to implement segmentation for porous materials in industrial tomographic images, resulting in superb quality results. Rashno et al. [22] developed a fully automated algorithm to segment fluid-filled and cyst regions in optical coherence tomography (OCT) retina images, segmented by combining a neutrosophic transformation and a graph-based shortest path method. Venhuizen et al. [23] developed a fully automatic segmentation system for segmenting retina in optical co- 
herence tomography (OCT) through using a Convolutional Neural Network (CNN), proving reliable efficiency. Similarly, another variant end-to-end Deep Deconvolutional Neural Network (DDNN) was presented by Men et al. [6] for multi-target segmentation in nasopharyngeal cancer.

Concerning practical MWT, Wang et al. [7] employed a comparative study on MWT image segmentation for breast cancer detection. They used a $K$-Nearest Neighbor (KNN) algorithm and Gaussian Mixture Model (GMM) in their research, and showed that KNN outperformed GMM when segmenting Region of Interest (RoI) when using the Mathew Correlation Coefficient (MCC). Another concrete comparative study was presented by Mahmood et al. [5], who demonstrated that MWT image segmentation is even capable of ameliorating the accuracy of image reconstruction using several automated segmentation methods. Joseph et al. [24] integrated image segmentation with their inverse scattering algorithm called Forward- Backward Time-Stepping (FBTS) to apply on microwave imaging for brain tumors. From the retrospective work, we determine to deploy two well-known approaches as pre-study to support our methodology.

\subsection{Otsu Algorithm}

The Otsu algorithm is an unsupervised method which can be used to select a threshold automatically from a gray level histogram and deal with the problem of selecting the thresholds in images [16]. This method plays an important role in the image segmentation because of its advantages of simple implement and time performance [25] such as the documented image segmentation based on the Otsu method $[26,15]$ and infrared image segmentation based on the improved Otsu method [27]. It is a widespread methodology used in dividing images into two categories of pixels; foreground and background. In this paper, this method is employed to segment the MWT image into two subcategories according to different levels of moisture. The description of how to segment the MWT image using the Otsu algorithm is explained as follows:

1. Calculate the histogram and probability of each intensity level.

2. Set up the initial $\omega_{i}(0)$ and $u_{i}(0)$.

3. Traverse the threshold values from $\mathrm{t}=155$ to 255 , update $\omega_{i}$ and $u_{i}$, and compute the variance $\sigma_{b}^{2}$.

The segmentation threshold of the different moisture levels is denoted by $\mathrm{t}$. The proportion of pixels belonging to a particular moisture level of the MWT image is recorded as $\omega_{0}(t)$, with the average gradation of $\mu_{0}(t)$. Moreover, the ratio of pixels in another moisture level is $\omega_{1}(t)$, and has an average gradation of $\mu_{1}(t)$. The average gradation of the entire MWT image is denoted by $\mu(\mathrm{t})$, the variance between classes is annotated by $\sigma_{\mathrm{b}}^{2}$. Assuming the size of MWT image is M (width) $* \mathrm{~N}$ (height), the number of pixels in which the gray value is lower than the threshold $\mathrm{T}$ in the corresponding image is $\mathrm{N}_{0}$. The number of pixels in which the gray value is above the threshold $\mathrm{T}$ is $\mathrm{N}_{1}$. Thus, we have:

$$
\begin{gathered}
\omega_{0}=\frac{N_{0}}{M * N} \\
\omega_{1}=\frac{N_{1}}{M * N} \\
N_{0}+N_{l}=M * N \\
\omega_{0}+\omega_{I}=1 \\
\mu=\omega_{0}^{*} \mu_{0}+\omega_{1} * \mu_{1} \\
\sigma_{b}^{2}=\omega_{0}\left(\mu_{0}-\mu\right)^{2}+\omega_{1}\left(\mu_{1}-\mu\right)^{2} \\
\sigma_{b}^{2}=\omega_{0} \omega_{0}\left(\mu_{0}-\mu_{1}\right)^{2}
\end{gathered}
$$

The boundaries between the low moisture areas and other areas will be found due to the values in these two sets of pixels through MWT images.

\section{$2.2 K$-means Algorithm}

The $K$-means algorithm is a method of cluster analysis in data mining [28] which can also be used in image segmentation. This genre of unsupervised machine learning algorithm aims to minimize the sum of squared distances between all points and the cluster center $[29,30]$. K denotes the number of centers we choose depending on the number of categories we intend to classify. In image segmentation, the $K$-means algorithm will help cluster images based on color and the minimum square Euclidean distance. In our study, this method is simultaneously used to find the boundary between the low moisture and other areas in MWT images. The value of $\mathrm{K}$ is chosen as two, as expected.

MWT pixel values range from 0 to 255 ; the size of the image is $\mathrm{M}$ (width) $* \mathrm{~N}$ (height). Assuming that the matrix of pixels in this image is $\left[\mathrm{X}_{11}, \mathrm{X}_{12}, \ldots, \mathrm{X}_{\mathrm{M}-1, \mathrm{~N}}\right.$, $X_{M, N}$ ] while the value of $K$ is two, the total pixel values in this matrix will be divided into two parts randomly. The first sets are $S_{1}=\left[W_{1}, W_{2}, \ldots, W_{N}\right]$ and the second sets are $S_{2}=\left[V_{1}, V_{2}, \ldots, V_{M}\right]$ separately. The overview and basic schematics of how the $K$-means method works in our study are elaborated as below: 
1. Choose two samples randomly from the dataset $\mathrm{D}=\left[\mathrm{X}_{11}, \mathrm{X}_{12}, \ldots, \mathrm{X}_{\mathrm{M}-1, \mathrm{~N}}, \mathrm{X}_{\mathrm{M}, \mathrm{N}}\right]$ as the initial centroid vector: $\mathrm{u}_{1}, \mathrm{u}_{2}$ and the first set is $\mathrm{S}_{1}$ and the another set is $\mathrm{S}_{2}$.

2. Calculate the distance between the element of dataset $x_{i}$ from first set $S_{1}$ and second set $S_{2}$ and the centroid $\mathrm{u}_{\mathrm{j}}: \mathrm{d}_{\mathrm{ij}}=\left\|\mathrm{x}_{\mathrm{i}}-\mathrm{u}_{\mathrm{j}}\right\|^{2}$. If $\mathrm{d}_{\mathrm{i} 1}>\mathrm{d}_{\mathrm{i} 2}, \mathrm{x}_{\mathrm{i}}$ will belong to $S_{1}$ otherwise $x_{i}$ will belong to $S_{2}$.

3. Output the classification $S=S_{1}, S_{2}$.

The boundaries between different levels of moisture will be found according to the values in these two sets of pixels in the MWT image.

\subsection{Performance Evaluation}

The accuracy is chosen for gauging the performance in this paper. To evaluate the accuracy obtained from our segmentation algorithms, the similarity and false positive are measured by jointly applying three criteria: Jaccard index, Dice coefficient and false positive:

- Jaccard index: This is a commonly used metric to derive the similarity and diversity of finite sample sets. We choose this statistic to gauge our study as it has been extensively applied in the context of image segmentation $[3,18]$. In our paper, it indicates the pixel-level similarity of input and output image. The input MWT image is referred as ground-truth while the output image represents the segmented image. The mathematical form of the Jaccard index is defined as (In this article, $G$ and $S$ annotate the input ground-truth image and the output segmented image respectively amid this denotation [18].):

$$
\text { Jaccard index }=\frac{|G \cap S|}{|G \cup S|}
$$

- Dice coefficient: This is another well-known parameter to measure the similarity between two samples. In our case, it will also result in pixel-level similarity between input and output. It differs from the Jaccard index which only calculate true positives once. The Dice coefficient normalizes the number of true positives to the average size of the two segmented areas [3]. It's defined as:

$$
\text { Dice coefficient }=2 \times \frac{|G| \cap|S|}{|G|+|S|}
$$

- False positive: This is the expectancy of the false positive ratio. This rate is the ratio between the number of negative samples falsely classified as positive and the total number of negative samples. Low values indicate high accuracy. A false positive manifests in the form of:

$$
\text { False positive }=\frac{|S|-|G \cap S|}{|G|}
$$

The performance metrics yield values ranging from zero to one. Reaching higher Jaccard and Dice values close to one represents a desirable segmentation result with high accuracy while a value approaching to zero of false positives achieves nearly perfect segmentation performance.

\subsection{Otsu VS $K$-means}

The designed pre-study is to compare the functionality between Otsu and $K$-means. Hence, we implement the same segmentation principles by using these two renowned approaches with our total 30 MWT images. Due to the limited space and better interpretation, we randomly select four samples as presentation. The outcomes are shown in Figure 2. In addition, the metric-driven quantitative evaluation among Jaccard index, Dice coefficient, and false positive is illustrated in Figures 3-5. From the results obtained, it is noteworthy that the $K$-means evidently outperforms Otsu method in most samples. Therefore, this inspiration enables us to develop our proposed state-of-the-art algorithm.

\section{METHOD}

This section presents our proposed algorithm while Otsu and $K$-means algorithms are chosen for the comparative evaluation of the proposed algorithm in terms of segmentation performance because of their proven reliability and success over the past few years.

\subsection{Overview of MWTS-KM}

We have demonstrated that $K$-means exceeds Otsu in our context from the conventional methods' perspective. The $K$-means algorithm is the one of the simplest clustering algorithms and is computationally faster than the hierarchical clustering. It can also work for a large number of variables. [2]. As a type of unsupervised machine learning algorithm, it is widely used in the realm of image segmentation due to its simplicity and efficiency. Many researchers have tried to combine it with other techniques to increase its performance, such as integrating with fuzzy c-means algorithms [4] and partial stretching enhancement [2]. Following such a strategy of combination, our proposed algorithm-MWT Segmentation based on $K$-means (MWTS-KM) - is established based on the basic $K$-means algorithm. It integrates image augmentation, grayscale image conversion and conventional $K$-means into our proposed automatic algorithm. This integrative strategy of our method is 

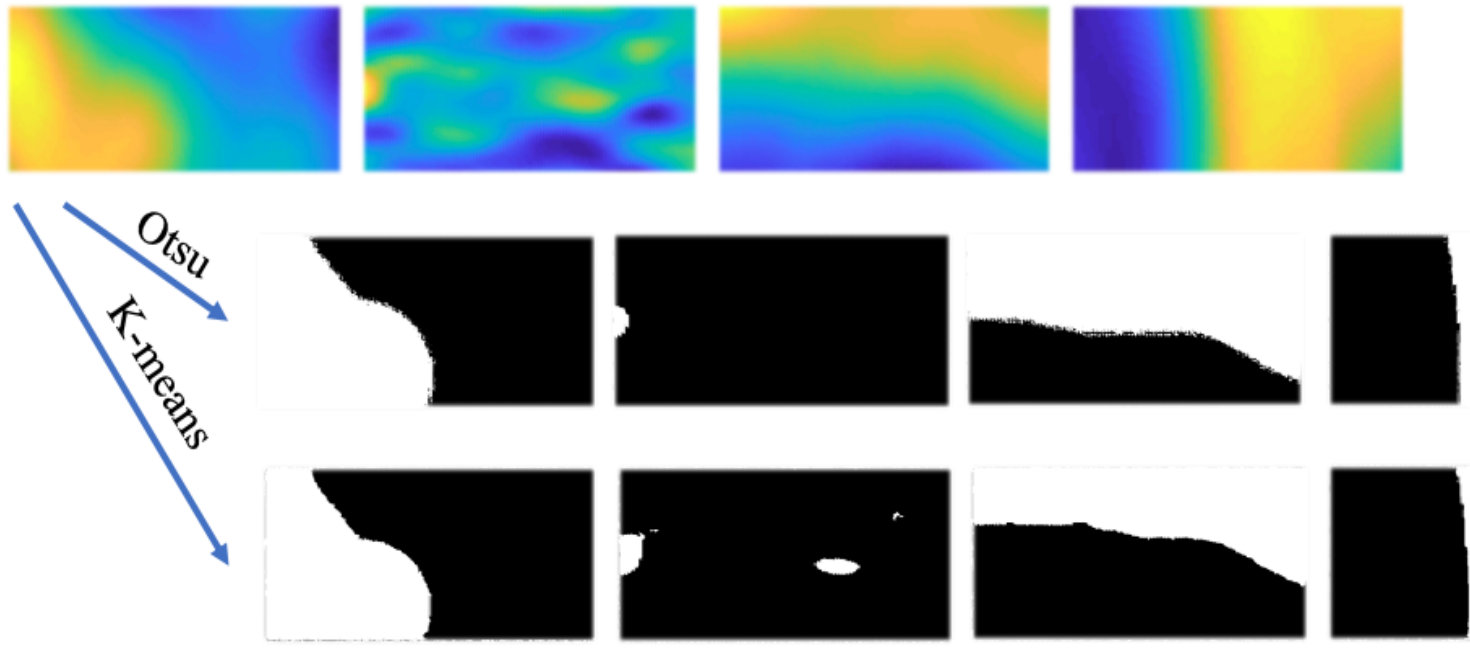

Figure 2: The comparison among Otsu and $K$-means. The first row shows the input images. The second row results from applying the Otsu algorithm on the input images while the third row results from $K$-means Algorithm.

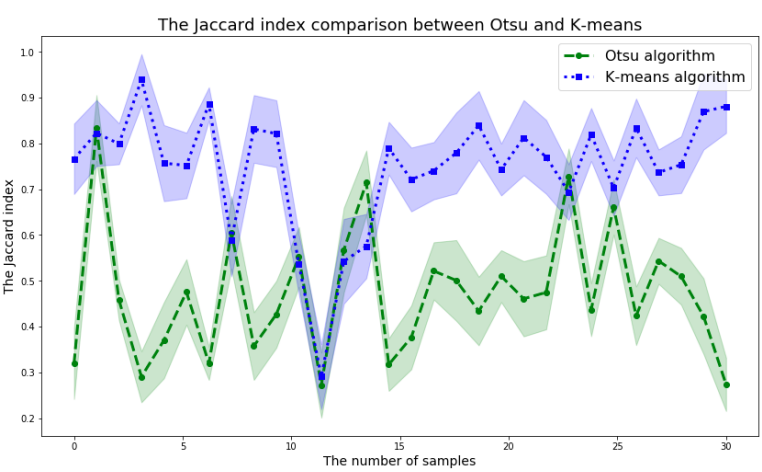

Figure 3: Jaccard index ( $0=$ low to $1=$ high) evaluation of Otsu and $K$-means algorithms for our 30 MWT images.

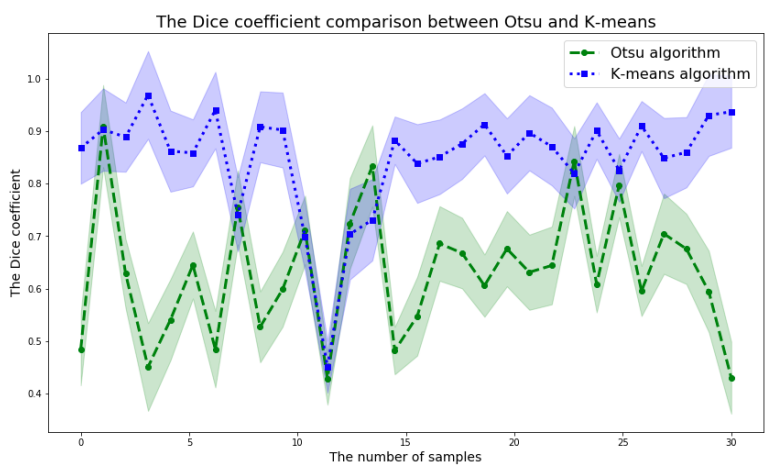

Figure 4: Dice coefficient ( $0=$ low to $1=$ high) evaluation of Otsu and $K$-means algorithms for our $30 \mathrm{MWT}$ images.

derived after considerable and objective test cases. The pipeline diagram shown in Figure 6 illustrates its structure and components; the pipeline stages 2, 3, and 4 are explained next.

\subsection{Image augmentation (Stage 2)}

The purpose of this step is to enhance the contrast and detect the boundaries displayed in our MWT images for

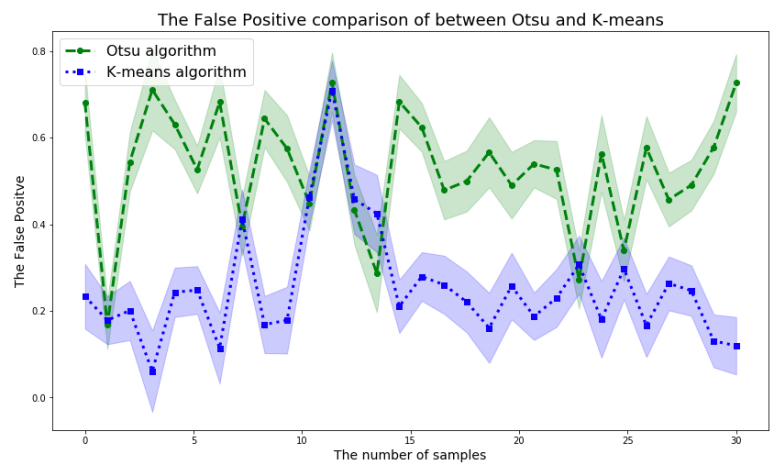

Figure 5: False positive ( $0=$ high to $1=$ low) evaluation of Otsu and $K$-means algorithms for our $30 \mathrm{MWT}$ images.

the foams we use. Image sharpening and removing blur as well as reducing noise are common image processing tasks [19]. After repeated trials, we find that simply using the contrast adjustment function with the same level factor for all images greatly helped segmentation with k-means later. The edges become sharper and with much less noise. Basically, this operation highlights the boundaries of different color pixels which represent different levels of moisture pertaining to foams. The contrast enhancement can be easily observed when used in grayscale conversion.

\subsection{Grayscale conversion (Stage 3)}

Grayscale images are distinct from purely black and white images in that they can represent several shades of gray in between. They are frequently used where each pixel is a single sample indicating intensity information only. A great number of segmentation methods have been successfully implemented in grayscale rather than ordinary RGB images in tomographic systems [1]. The segmentation contributes the desirable consequences through grayscale tomographic 


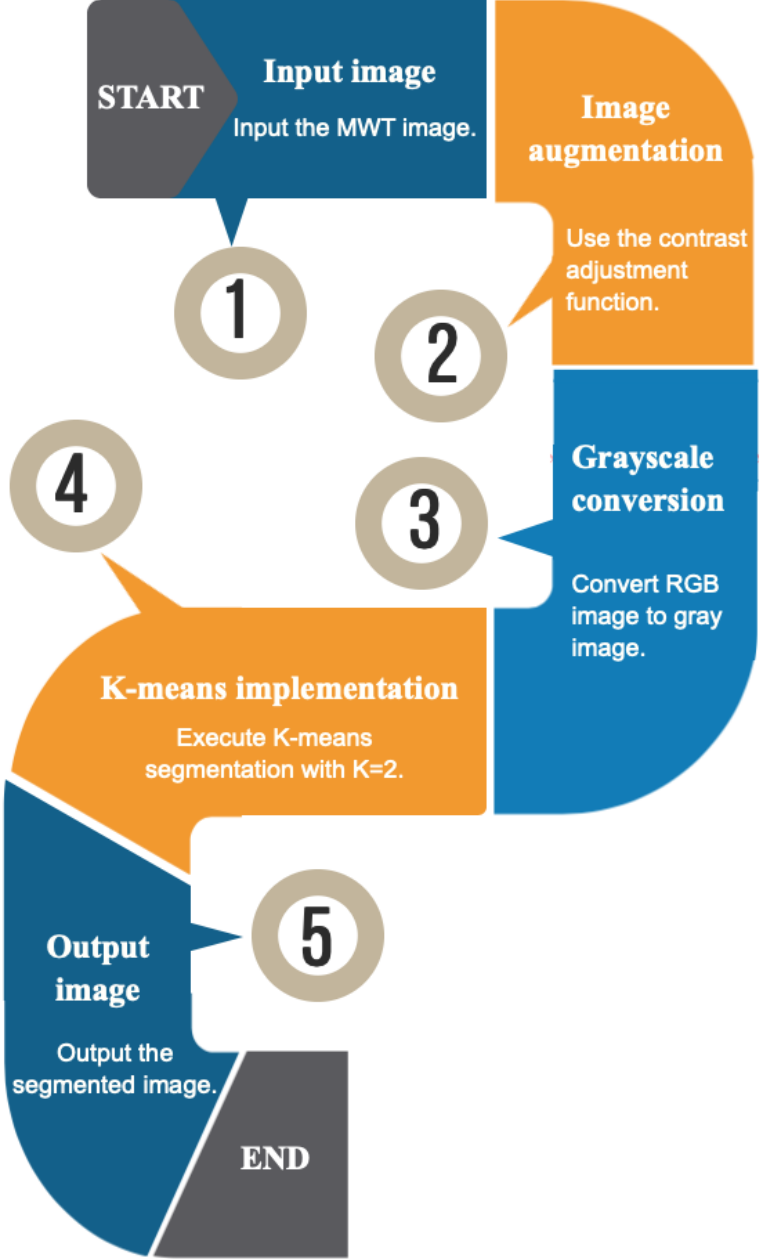

Figure 6: The pipeline of proposed MWT Segmentation based on $K$-means (MWTS-KM) algorithm. (Step 2 and step 3 are explained in Figure 8)

images while segmenting for porous materials [19].We use the Python Imaging Library (PIL) to execute the grayscale conversion. The comparison between the input MWT and grayscale images (after contrast adjustment/augmentation), and between just contrast adjustment/augmentation and grayscale applied afterwards are displayed in Figure 8.

\section{4 $K$-means implementation (Stage 4)}

A $K$-means algorithm is used as the final step of our proposed algorithm to execute the segmentation stage. A fuller introduction in the following section describes how the conventional $K$-means algorithm is used in this paper for the purpose of comparison.

\section{SETUP AND RESULTS}

Microwave tomography mainly consists of two steps: collecting scattered electric field data around an object, and applying inversion algorithms on the scattered field data to get information of the object properties like dielectric distribution. As for the setup, the object under testing is surrounded by an array of antennas acting as transceivers. The scattered field data is collected so that each antenna is separate in the source mode, and it is assumed that it cannot receive data while acting as a source. A schematic picture proposed for the MWT is shown in Figure 7, where the antenna array is modelled as 2D line sources and a perfectly matched layer is placed over the truncated free-space.

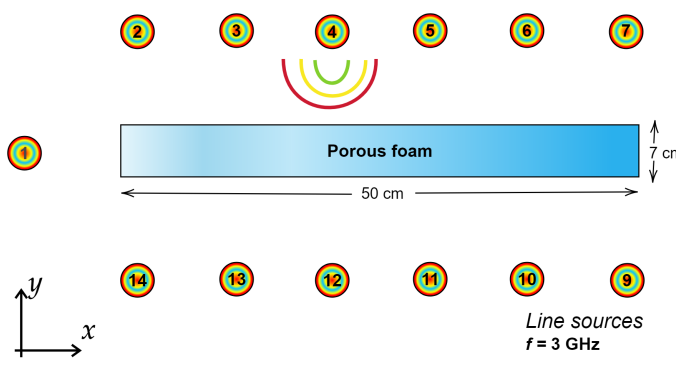

Figure 7: Model used for benchmarking purposes. The light blue box is the porous foam; green circles represent the antenna modelled as a 2D line source.

Here, the object is a piece of porous foams. Detailing the reconstruction method for estimating the moisture distribution is beyond the scope of this paper. Instead, we study automatic segmentation methodologies for the purpose of visualizing the low moisture area displayed in the MWT reconstructed images. In this study, we gather a total of 30 MWT images reconstructed from the data obtained from the sensors characterizing 30 distinct processes. The reconstructed images are all in RGB mode, and their size are kept constant throughout augmentation, grayscale conversion and three distinct segmentation phases.

\subsection{Results}

We conduct segmentation in all the 30 images gained from different microwave drying processes as aforementioned. In our analysis, the segmentation task is a binary classification to distinguish the foreground and the background in images. Accordingly, we intend to categorize our MWT image into two parts; low moisture area and other area. The whole segmentation period for 30 images lasts around three hours. We run all the python scripts in the full-fledged IDE-Jupyter Notebook. As aforementioned, we randomly choose 4 of those 30 samples to be shown in this paper (Figures 8 and 9) for readability and understanding due to the given limited space. The blue areas in the MWT images represent low moisture areas. Figure 8 shows a part of the pipeline of MWTS-KM, comparing the input MWT images, augmented MWT images and grayscale MWT images in which it is easy to differentiate low moisture 

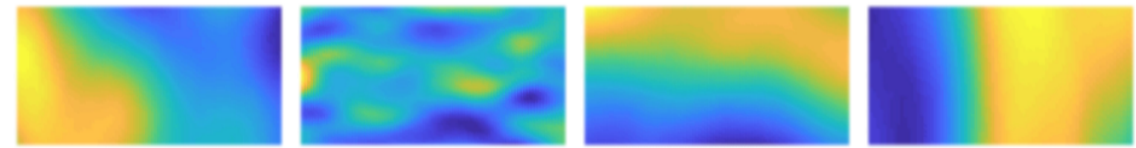

\section{Input MWT images}

\section{Augmented MWT images}
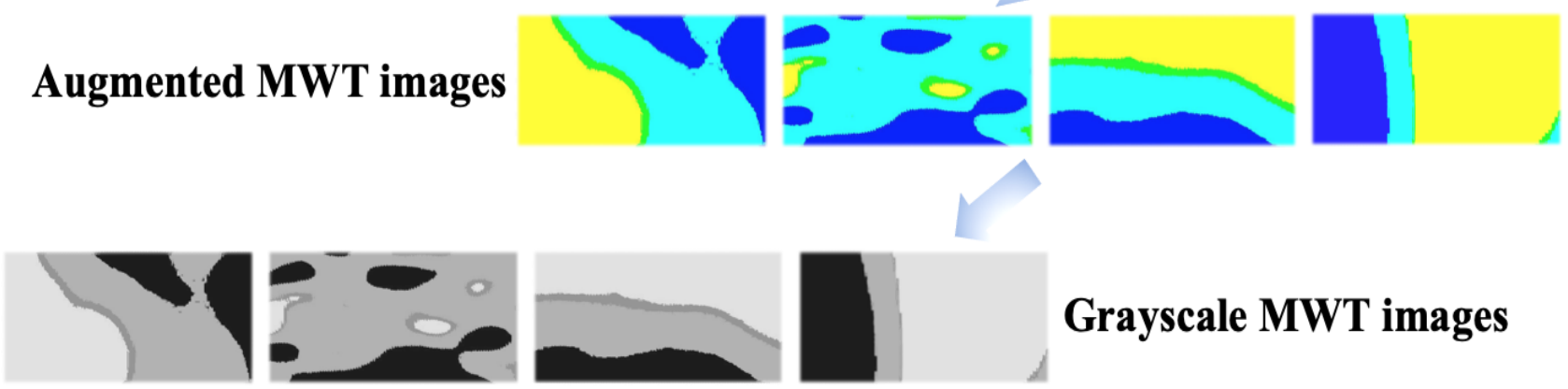

\section{Grayscale MWT images}

Figure 8: The comparison among input MWT images, augmented images and grayscale images. The first row stands for input MWT image, second row for augmented images and third row for grayscale images.
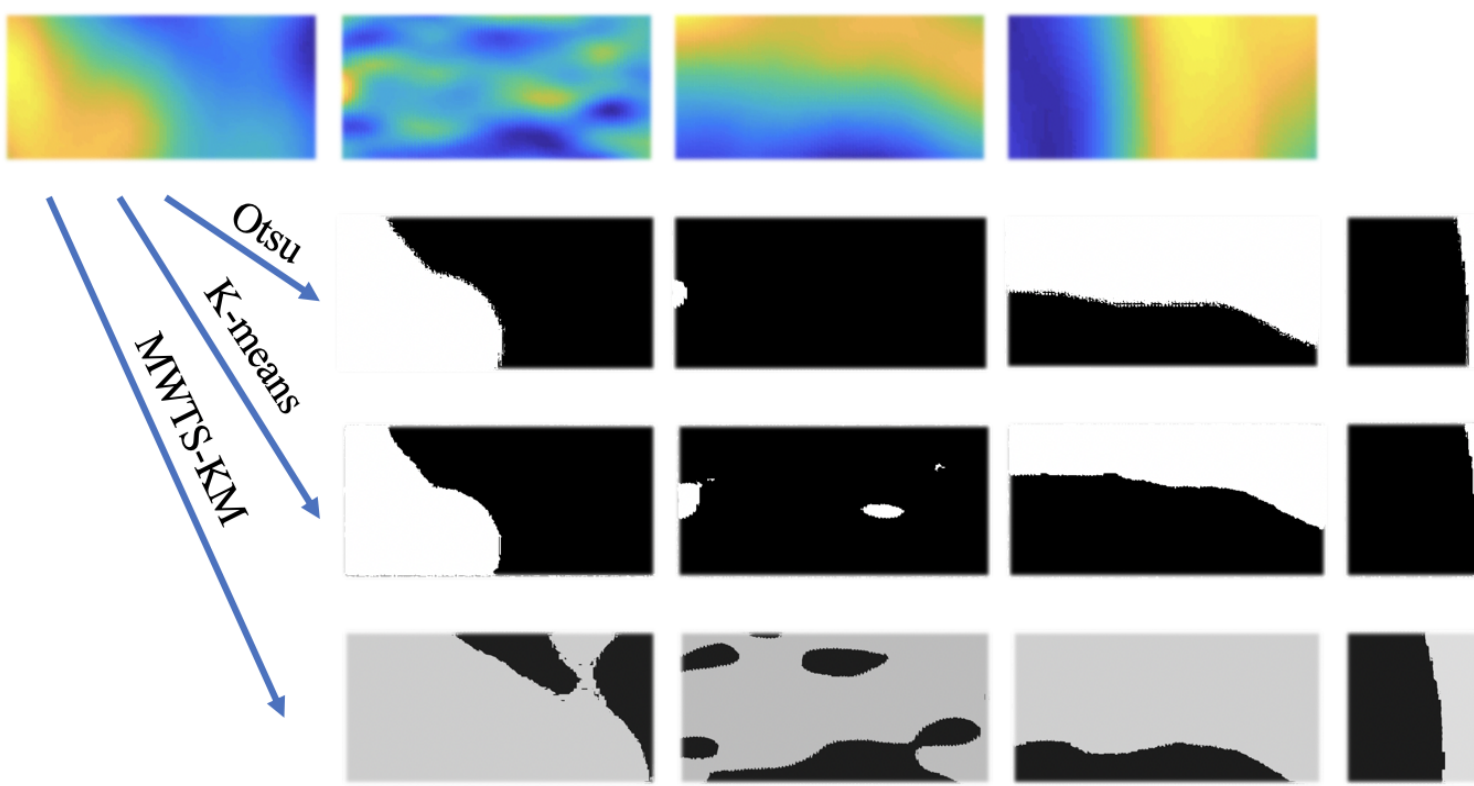

Figure 9: The comparison among three segmentation results. The first row shows the input images. The second row results from applying the Otsu algorithm on the input images. The third row results from using conventional $K$-means Algorithm while the last row shows output images from the MWTS-KM algorithm.

areas and other areas. The exhaustive segmentation results are shown in Figure 8 with the respect of MWTS$\mathrm{KM}$, Otsu and $K$-means algorithms. Dark areas in these images denote the low moisture areas that are the desirable consequences we want.

\subsection{Evaluation}

As Figure 9 depicts, the Otsu and $K$-means algorithms are not capable to visualize the low moisture areas after segmentation while MWTS-KM precisely visualized those designated areas. More specifically, there is almost no evident difference observed between the segmentation results from Otsu and $K$-means algorithms, both of which are ineffective in obtaining desirable consequences. However, compared to ground-truth images which are input MWT images, our proposed MWTS-KM achieves excellent accuracy in visualizing low moisture areas in terms of perception. Therefore, we provide another more convincing quantitative evaluation.

The complete quantitative evaluation output for the 30 samples is shown in Table 1, presenting the Jaccard index, Dice coefficient and false positive across all three algorithms. In terms of performance of each algorithm, MWTS-KM exceeds Otsu and $K$-means remarkably in each metric. While $K$-means performs better than Otsu in most cases, it is outperformed by MWTS-KM. In terms of the Jaccard index, Otsu produces values fluc- 
Table 1: Jaccard index, Dice coefficient, false positive: Comprehensive performance comparison between 3 segmentation algorithms for 30 samples.

\begin{tabular}{|c|c|c|c|c|c|c|c|c|c|}
\hline \multicolumn{3}{|c|}{ Jaccard index } & \multicolumn{3}{|c|}{ Dice coefficient } & \multicolumn{3}{|c|}{ False positive } & \multirow[b]{2}{*}{$K$-means } \\
\hline & MWTS-KM & Otsu & $K$-means & MWTS-KM & Otsu & $K$-means & MWTS-KM & Otsu & \\
\hline (1) & 0.997 & 0.319 & 0.766 & 0.999 & 0.484 & 0.868 & 0.003 & 0.682 & 0.234 \\
\hline (2) & 0.998 & 0.834 & 0.823 & 0.999 & 0.909 & 0.903 & 0.002 & 0.168 & 0.178 \\
\hline (3) & 0.995 & 0.458 & 0.799 & 0.997 & 0.628 & 0.888 & 0.005 & 0.544 & 0.201 \\
\hline (4) & 0.995 & 0.291 & 0.939 & 0.998 & 0.450 & 0.969 & 0.005 & 0.711 & 0.061 \\
\hline (5) & 0.997 & 0.370 & 0.757 & 0.998 & 0.540 & 0.862 & 0.003 & 0.631 & 0.243 \\
\hline (6) & 0.995 & 0.476 & 0.752 & 0.998 & 0.645 & 0.858 & 0.005 & 0.527 & 0.248 \\
\hline (7) & 0.994 & 0.320 & 0.887 & 0.997 & 0.484 & 0.940 & 0.006 & 0.682 & 0.114 \\
\hline (8) & 0.998 & 0.606 & 0.589 & 0.999 & 0.754 & 0.741 & 0.002 & 0.395 & 0.412 \\
\hline (9) & 0.995 & 0.358 & 0.832 & 0.998 & 0.527 & 0.908 & 0.005 & 0.644 & 0.168 \\
\hline (10) & 0.995 & 0.427 & 0.821 & 0.998 & 0.599 & 0.902 & 0.005 & 0.575 & 0.179 \\
\hline (11) & 0.999 & 0.552 & 0.536 & 0.999 & 0.712 & 0.698 & 0.001 & 0.448 & 0.464 \\
\hline (12) & 0.999 & 0.272 & 0.291 & 0.999 & 0.428 & 0.451 & 0.001 & 0.728 & 0.709 \\
\hline (13) & 0.999 & 0.567 & 0.543 & 0.999 & 0.724 & 0.704 & 0.001 & 0.433 & 0.458 \\
\hline (14) & 0.999 & 0.714 & 0.576 & 0.999 & 0.833 & 0.731 & 0.001 & 0.286 & 0.425 \\
\hline (15) & 0.999 & 0.317 & 0.790 & 0.999 & 0.482 & 0.882 & 0.001 & 0.683 & 0.211 \\
\hline (16) & 0.999 & 0.377 & 0.721 & 0.999 & 0.547 & 0.838 & 0.001 & 0.624 & 0.279 \\
\hline (17) & 0.999 & 0.522 & 0.740 & 0.999 & 0.686 & 0.851 & 0.001 & 0.479 & 0.260 \\
\hline (18) & 0.999 & 0.501 & 0.779 & 0.998 & 0.667 & 0.876 & 0.002 & 0.500 & 0.221 \\
\hline (19) & 0.999 & 0.434 & 0.840 & 0.998 & 0.605 & 0.913 & 0.001 & 0.566 & 0.161 \\
\hline (20) & 0.999 & 0.510 & 0.743 & 0.998 & 0.676 & 0.853 & 0.002 & 0.490 & 0.257 \\
\hline (21) & 0.999 & 0.461 & 0.813 & 0.999 & 0.631 & 0.897 & 0.001 & 0.540 & 0.187 \\
\hline (22) & 0.999 & 0.475 & 0.771 & 0.997 & 0.644 & 0.870 & 0.001 & 0.526 & 0.230 \\
\hline (23) & 0.999 & 0.728 & 0.694 & 0.998 & 0.843 & 0.819 & 0.001 & 0.272 & 0.307 \\
\hline (24) & 0.999 & 0.437 & 0.819 & 0.999 & 0.608 & 0.901 & 0.001 & 0.563 & 0.181 \\
\hline (25) & 0.999 & 0.661 & 0.703 & 0.999 & 0.796 & 0.825 & 0.001 & 0.339 & 0.297 \\
\hline 26 & 0.998 & 0.424 & 0.834 & 0.999 & 0.595 & 0.909 & 0.001 & 0.577 & 0.166 \\
\hline (27) & 0.997 & 0.544 & 0.737 & 0.998 & 0.704 & 0.848 & 0.001 & 0.457 & 0.263 \\
\hline (28) & 0.999 & 0.510 & 0.754 & 0.998 & 0.675 & 0.860 & 0.001 & 0.490 & 0.247 \\
\hline (29) & 0.999 & 0.423 & 0.869 & 0.997 & 0.594 & 0.930 & 0.002 & 0.578 & 0.131 \\
\hline (30) & 0.998 & 0.274 & 0.881 & 0.995 & 0.430 & 0.937 & 0.001 & 0.727 & 0.119 \\
\hline
\end{tabular}

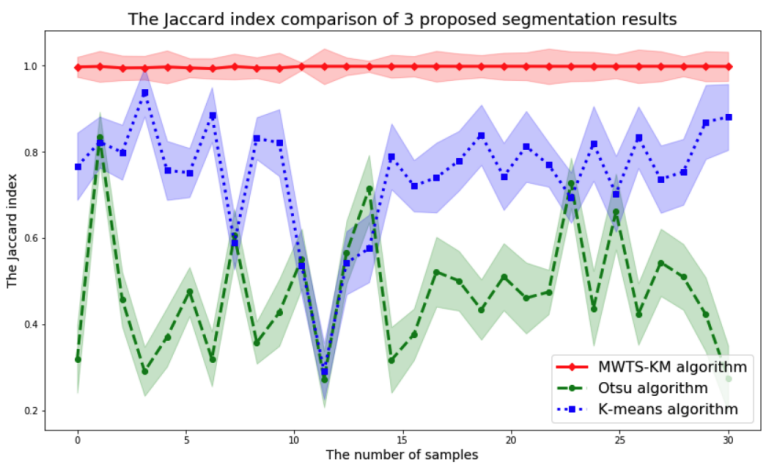

Figure 10: Jaccard index accuracy ( $0=$ low to $1=$ high) evaluation of MWTS-KM, Otsu, $K$-means segmentation algorithms for tested 30 MWT images.

tuating around 0.50 for 30 samples while $K$-means produces a cluster of values around 0.80. Similarly, Otsu obtains these Dice coefficients oscillating close to 0.60

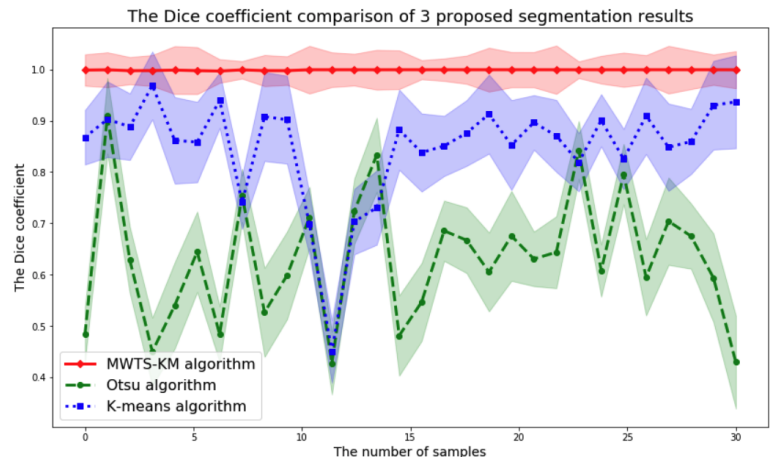

Figure 11: Dice coefficient accuracy ( $0=$ low to $1=$ high) evaluation of MWTS-KM, Otsu, $K$-means segmentation algorithms for test 30 images.

but $K$-means has a series of Dice values in proximity of about 0.82 . When considering false positive, Otsu always has a higher value compared to $K$-means, which 


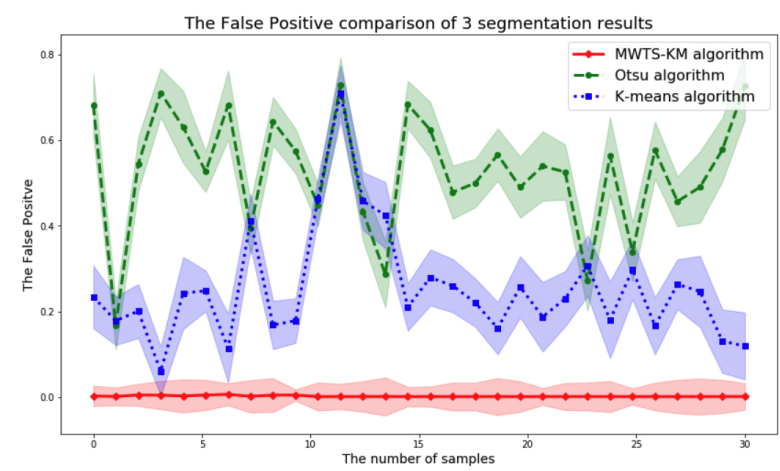

Figure 12: False positive ( $0=$ high to $1=$ low) evaluation of MWTS-KM, Otsu, $K$-means segmentation algorithms for test 30 images.

also confirms the above results. The visual and straightforward comparisons are displayed in Figures 10, 11, and 12 .

MWTS-KM in particular outperforms Otsu and $K$-means exceedingly according to our sensitivity analysis. In fact, Otsu even has superior performance compared to $K$-means in a few experimental cases in line with quantitative evaluation. Overall, these two well-established approaches are disadvantageous in our context. It is worthwhile to observe that MWTS-KM achieves nearly one of both Jaccard index and Dice coefficient in the whole 30 segmentation results. Furthermore, the false positive values for MWTS-KM attain almost zero in each trial, which would be pertained to exceptional performance.

\section{DISCUSSIONS}

To summarize, we implement image segmentation technology in 30 MWT images obtained from the specialized microwave drying processes. The proposed MWTS-KM algorithm is applied to segment images to visualize the areas indicating low moisture level for porous foams in relevant context. We compare MWTS$\mathrm{KM}$ with another two commonly used methods and appraise them thoroughly. $K$-means algorithm surpasses Otsu in general cases even though there is only a moderate difference between them. As shown in Figures 9, 10 and 11 , there exists some intersections among Otsu and $K$-means in their performance line-plots. For example, in samples 12, 13 and 23, the Otsu algorithm is superior to $K$-means algorithm whereas it is disadvantageous compared to $K$-means algorithm in other samples. However, the red lines representing MWTS$\mathrm{KM}$ indicate that our proposed algorithm has the highest Jaccard indexes and Dice coefficients with the lowest false positive values as a tremendously acceptable result.

Overall, our proposed MWTS-KM algorithm performs better than Otsu and $K$-means algorithms in both perceived and quantitative evaluation in MWT image seg- mentation, especially outperforming them dominantly in each case we experimented.

\section{CONCLUSIONS}

In this paper, firstly we prove the mature segmentation methods which have been widely used in tomographic systems are considerably suitable for MWT image segmentation. In our study, this technique is an intuitive and innovative method for visualizing the low moisture areas of foams. We have developed an entirely automatic methodology named MWTS-KM to conduct the MWT image segmentation, validating its high efficiency and high accuracy after practical experiments. This method is able to meticulously visualize the low moisture areas for foams in MWT images. Furthermore, its performance is superior to two other preeminent methods.

We acknowledge that there still are some inefficiencies in our algorithm, such as inadequate samples and lack of more complete pre-processing steps. Therefore, future work will mainly concentrate on adding more preprocessing techniques as well as testing more sample trials to enhance the robustness of the algorithm. For instance, thresholding could be a promising add-on. Additionally, we will incorporate human perception into the study, such as by changing the color schemes to compare the task performance.

\section{ACKNOWLEDGMENTS}

This project has received funding from the European Union's Horizon 2020 research and innovation programme under the Marie Sklodowska-Curie grant agreement No. 764902.

\section{REFERENCES}

[1] O. M. Dorgham, "Automatic body segmentation from computed tomography image," in 2017 International Conference on Advanced Technologies for Signal and Image Processing (ATSIP), pp. 1-5, May 2017.

[2] N. Dhanachandra, K. Manglem, and Y. J. Chanu, "Image segmentation using k-means clustering algorithm and subtractive clustering algorithm," Procedia Computer Science, vol. 54, pp. 764-771, 2015.

[3] B. H. Menze, A. Jakab, S. Bauer, J. KalpathyCramer, K. Farahani, J. Kirby, Y. Burren, N. Porz, J. Slotboom, R. Wiest, et al., "The multimodal brain tumor image segmentation benchmark (brats)," IEEE transactions on medical imaging, vol. 34, no. 10, pp. 1993-2024, 2014.

[4] A. Jose, S.Ravi, and M.Sambath, "Brain tumor segmentation using k-means clustering and fuzzy c-means algorithms and its area calculation," 
International Journal of Innovative Research in Computer and Communication Engineering, vol. 2, pp. 1-9, Mar 2014.

[5] Q. Mahmood, S. Li, A. Fhager, S. Candefjord, A. Chodorowski, A. Mehnert, and M. Persson, "A comparative study of automated segmentation methods for use in a microwave tomography system for imaging intracerebral hemorrhage in stroke patients," Journal of Electromagnetic Analysis and Applications, vol. 7, no. 05, p. 152, 2015.

[6] K. Men, X. Chen, Y. Zhang, T. Zhang, J. Dai, J. Yi, and Y. Li, "Deep deconvolutional neural network for target segmentation of nasopharyngeal cancer in planning computed tomography images," Frontiers in oncology, vol. 7, p. 315, 2017.

[7] C. Wang, W. Wang, S. Shin, and S. I. Jeon, "Comparative study of microwave tomography segmentation techniques based on gmm and knn in breast cancer detection," in Proceedings of the 2014 Conference on Research in Adaptive and Convergent Systems, pp. 303-308, ACM, 2014.

[8] P. Mojabi, M. Ostadrahimi, L. Shafai, and J. LoVetri, "Microwave tomography techniques and algorithms: A review," in 201215 International Symposium on Antenna Technology and Applied Electromagnetics, pp. 1-4, June 2012.

[9] Z. Wu and H. Wang, "Microwave tomography for industrial process imaging: Example applications and experimental results.," IEEE Antennas and Propagation Magazine, vol. 59, pp. 61-71, Oct 2017.

[10] A. Fhager, M. Gustafsson, and S. Nordebo, "Image reconstruction in microwave tomography using a dielectric debye model," IEEE Transactions on Biomedical Engineering, vol. 59, pp. 156-166, Jan 2012.

[11] M. Pastorino, Microwave imaging, vol. 208. John Wiley \& Sons, 2010.

[12] A. H. Sihvola, Electromagnetic mixing formulas and applications. No. 47, Iet, 1999.

[13] Y. Zhang, Y. Ma, A. Omrani, R. Yadav, M. Fjeld, and M. Fratarcangeli, "Automatic image segmentation for microwave tomography (mwt) from implementation to comparative evaluation," in Proceedings of the 12th International Symposium on Visual Information Communication and Interaction, pp. 1-2, 2019.

[14] S. Neeraj and L. M. Aggarwal, "Automated medical image segmentation techniques," J Med Phys, vol. 35, pp. 3-14, Jan-Mar 2010.

[15] M. Shoaib, R. Naseem, and A. H. Dar, "Automated segmentation of lungs incomputed tomographic images," European Journal of Scientific
Research, vol. 98, pp. 45-54, Mar 2013.

[16] N. Otsu, "A threshold selection method from graylevel histograms," IEEE transactions on systems, man, and cybernetics, vol. 9, no. 1, pp. 62-66, 1979.

[17] H. Permuter, J. Francos, and I. Jermyn, "A study of gaussian mixture models of color and texture features for image classification and segmentation," Pattern Recognition, vol. 39, no. 4, pp. 695 - 706, 2006. Graph-based Representations.

[18] S. Wu, S. Yu, L. Zhuang, X. Wei, M. Sak, N. Duric, J. Hu, and Y. Xie, "Automatic segmentation of ultrasound tomography image," BioMed research international, vol. 2017, 2017.

[19] A. P. Sheppard, R. M. Sok, and H. Averdunk, "Techniques for image enhancement and segmentation of tomographic images of porous materials," Physica A: Statistical Mechanics and its Applications, vol. 339, no. 1, pp. 145 - 151, 2004. Proceedings of the International Conference New Materials and Complexity.

[20] M. Kass, A. Witkin, and D. Terzopoulos, "Snakes: Active contour models," International Journal of Computer Vision, vol. 1, pp. 321-331, Jan 1988.

[21] L. Vincent and P. Soille, "Watersheds in digital spaces: An e cient algorithm based on immersion simulations," IEEE Trans. on Pattern Analysis and Machine Intelligence, vol. 13.

[22] A. Rashno, D. D. Koozekanani, P. M. Drayna, B. Nazari, S. Sadri, H. Rabbani, and K. K. Parhi, "Fully automated segmentation of fluid/cyst regions in optical coherence tomography images with diabetic macular edema using neutrosophic sets and graph algorithms," IEEE Transactions on Biomedical Engineering, vol. 65, no. 5, pp. 9891001, 2018.

[23] F. G. Venhuizen, B. van Ginneken, B. Liefers, M. J. van Grinsven, S. Fauser, C. Hoyng, T. Theelen, and C. I. Sánchez, "Robust total retina thickness segmentation in optical coherence tomography images using convolutional neural networks," Biomed. Opt. Express, vol. 8, pp. 3292-3316, Jul 2017.

[24] E. J. Joseph, K. A. H. Ping, K. Kipli, D. A. A. Mat, S. Sahrani, D. N. A. Zaidel, M. I. Sariphn, and M. H. Marhaban, "Integration of image segmentation method in inverse scattering for brain tumour detection," Progress In Electromagnetics Research, vol. 61, pp. 111-122, 2017.

[25] H. J. Vala and A. Baxi, "A review on otsu image segmentation algorithm," International Journal of Advanced Research in Computer Engineering \& Technology (IJARCET), vol. 2, no. 2, pp. 387389, 2013. 
[26] J. Zhang and J. Hu, "Image segmentation based on $2 \mathrm{~d}$ otsu method with histogram analysis," in 2008 International Conference on Computer Science and Software Engineering, vol. 6, pp. 105-108, IEEE, 2008.

[27] Y. Wang, "Improved otsu and adaptive genetic algorithm for infrared image segmentation," in 2018 Chinese Control And Decision Conference (CCDC), pp. 5644-5648, IEEE, 2018.

[28] J. A. Hartigan and M. A. Wong, "Algorithm as 136: A k-means clustering algorithm," Journal of the Royal Statistical Society. Series C (Applied Statistics), vol. 28, no. 1, pp. 100-108, 1979.

[29] S. Ray and R. H. Turi, "Determination of number of clusters in k-means clustering and application in colour image segmentation," in Proceedings of the 4th international conference on advances in pattern recognition and digital techniques, pp. 137-143, Calcutta, India, 1999.

[30] N. Sharma and L. M. Aggarwal, "Automated medical image segmentation techniques," Journal of medical physics/Association of Medical Physicists of India, vol. 35, no. 1, p. 3, 2010. 